\title{
The Validity of the Screen for Child Anxiety Related Emotional Disorders Revised (SCARED-R) Scale and Sub-Scales in Swedish Youth
}

\author{
Tord Ivarsson $^{1}$ (D) Gudmundur Skarphedinsson ${ }^{2} \cdot$ Markus Andersson $^{3,4}$. \\ Håkan Jarbin ${ }^{3,4}$
}

Published online: 29 July 2017

(C) The Author(s) 2017. This article is an open access publication

\begin{abstract}
We evaluated the clinical utility of the Swedish SCARED-R in child- and adolescent psychiatric outpatients $(n=239)$ and validated it against Longitudinal Expert All Data (LEAD) DSM IV diagnoses based on the Children's Schedule for Affective Disorders and Schizophrenia (KSADS) and subsequent clinical work-up and treatment outcome. The SCARED-R total score and subscales had acceptable sensitivity/specificity for child and parent reports for cut-offs based on Receiver Operating Characteristics (ROC) curves, with mostly moderate area under the curve. Sensitivity ranged from $75 \%$ (parent rated social anxiety) to $79 \%$ [child rated Generalized Anxiety Disorder (GAD)]. Specificity, ranged from $60 \%$ for child-rated GAD to $88 \%$ for parent rated social anxiety. Parent-child agreement was moderate, and each informant provided unique information contributing to most diagnoses. In conclusion, the SCARED-R is useful for screening anxiety symptoms
\end{abstract}

Tord Ivarsson

tord.ivarsson@r-bup.no

Gudmundur Skarphedinsson

gudmundr@gmail.com

Markus Andersson

markus.andersson@ regionhalland.se

Håkan Jarbin

Hakan.Jarbin@ @regionhalland.se

1 The Centre for Child and Adolescent Mental Health, Postboks 4623 Nydalen, 0405 Oslo, Norway

2 The Faculty of Psychology, University of Iceland, Reykjavík, Iceland

3 Department of Clinical Sciences, Child and Adolescent Psychiatry, Lund University, Lund, Sweden

4 Region Halland, Sweden. BUP, HSH, SE-301 85 Halmstad, Sweden in clinical populations. However, it cannot replace interview based diagnoses, nor is it adequate to use just one informant.

Keywords Self-rating scale Parent rating scale . Anxiety disorder $\cdot$ Concurrent validity $\cdot$ Discriminant validity $\cdot$ LEAD diagnosis

\section{Introduction}

Anxiety disorders are prevalent paediatric mental disorders $[1,2]$. In the short-term they are stable [3], and in the longterm they show both homo-typic [4] and hetero-typic continuity [5]. In the latter respect, childhood and adolescent anxiety disorders confer a higher risk of both depression and somatic illness, but also of future drug use [5]. Furthermore, anxiety disorders have a negative impact on various domains of functioning, e.g. at school, in social life, and in family relations [6].

Moreover, many types of burdens are associated with anxiety disorders, including increased costs associated with the illness both due to the child staying away from school and due to parents' leave from work [7]. Some of these effects are found even in long-term follow-up in adulthood, e.g. lower income, and difficulties in social relationships [5].

Despite these disorders' high prevalence in the general population, they are less common in child psychiatric care than their proportion in the general population would imply. Referrals to the specialized child- and adolescent psychiatry (CAP) services are greater for externalizing and disruptive disorders than for anxiety disorders. Gren Landell [8] found, for example, that just one out of five children with social anxiety disorder in a high school study had 
a CAP contact and Hansen [9] noted in a CAP outpatient sample that about half of those with a KSADS anxiety disorder at intake had not been referred because of anxiety (see as well [10, 11]). Similarly, Heyman [12] found in an epidemiological study 25 children with OCD $(0.25 \%$ of all children 6-17 years of age), and only three of them were known within the specialized CAP services, although a good third had consulted their general practitioner. Thus, there is a need for better screens for anxiety symptoms to be used in primary care and in the school health care.

Another problem is that children with anxiety disorders do not seek help themselves like adults do. They are dependent on parents or teachers to recognize the symptoms and the need. Thus, there is a question of how to evaluate and compare the information an informant provides, i.e., which information, when applied in a scale, is best fitted for aiding the diagnostic process [13]. There are several challenges associated with such an evaluation. With regard to parental reports of the symptoms of anxiety disorders, those that are overt, i.e. the disorders' behavioural manifestations, can be observed by the parent and may be easier to rate [14]. In these respects, the challenge concerns the interpretation of at least some of these overt behaviours. The interpretation is dependent on an understanding of the motivation underlying a behaviour or an adequate attribution of the cause of a behaviour [14]: is a refusal to go to school due to anxiety and an avoidance, or an expression of oppositionality with a wish to stay home and, for example, do more pleasurable activities? Thus, the level of parental insight into the child's thoughts and feelings are crucial, and this is even more important about symptoms that are covert. They need to be reported to, or told the parent, and then translated to the items in the parent scale.

Children are commonly regarded as the best informant on subjective aspects of the disorder [15]. However, the "mapping" of these symptoms, i.e., reported by the child on a child scale is not without problems. In younger children or developmentally delayed or disordered children, a lack of introspective capacities and language skills may compromise the reliability of these assessments. Moreover, children may be less exact with describing impairment, duration, and intensity/severity.

The use of multiple informants, including both a children's and a parental symptom assessment have, however, but a low to moderate agreement $[15,16]$. Thus, we are posed with the problems on how to combine the information. In general, children tend to report more severe anxiety levels than parents do $[15,17]$.

Theses discrepancies in self- and parental reports makes it important to evaluate the information from different informants on a disorder specific level as the disorders vary with regard to how much the subjective perspective is central, and to which extent behavioural manifestations are core features.

Several self- and parent rating scales for anxiety symptoms have been constructed, and need to be adjusted to the changing perspectives as the diagnostic systems evolve. Recently, Muris and an expert group published an anxiety scale developed for the DSM-5 [18-20]. The Screen for Child Anxiety Related Emotional Disorders which is evaluated in the paper and that was published by Birmaher [21] in 1997 covering the classical anxiety disorders (Separation anxiety, Social Anxiety and GAD) as well as panic disorder and school phobia was developed for the DSM IV [22]. Its psychometric properties were found to be good as well in a replication study [23] (see also [2, 3, 24]). Muris [25, 26] added some additional items to screen for OCD, specific Phobias (SP) and acute/post-traumatic stress disorder (A/ PTSD) as well. The revised scale is called the SCARED-R and its reliability and discriminant validity was studied further in a clinical sample [27]. Muris [27] found a satisfactory discriminant validity both in between the anxiety- and other disorders as well as within the group of anxiety disorders itself. He noted that the SCARED-R had a reasonable capacity to predict specific anxiety disorders. No normative SCARED or SCARED-R data have been published in Sweden. However, in Norway (with a culture close to Sweden) Leikanger $[2,3]$ used the original SCARED in a sizeable sample of adolescents and has published normative data, as well as data on symptom change across 1 year.

The SCARED-R was translated into Swedish in 2008 for use in a treatment project in paediatric OCD [28]. In the current paper, the utility of the Swedish SCARED/ SCARED-R is examined in a clinical diagnostic study [29] and psychometric data are published for the first time. In addition, the diagnostic accuracy is improved as we use KSADS diagnoses improved on through a LEAD process (see below) to validate the SCARED-R and its sub-scales. The advantage with the LEAD process is that additional information that may not be divulged in an interview setting may come to the foreground as the work-up and the treatment unfolds. Moreover, the level of impairment that each disorder entails is assessed not only at the intake assessments, but is supplemented through both structured and more informal contacts with the school that the LEAD procedure may consider.

\section{Aims}

To recommend cut-off scores for the SCARED-R based on ROC curves for each scale/sub-scale, and to investigate their usefulness in a psychiatric outpatient population based on the psychometric properties. The SCARED-Rs 
concurrent and discriminant validity against the LEAD diagnoses are examined.

\section{Methods}

\section{Subjects}

In all we included 307 CAP outpatients, who consecutively (from January 2010 to March 2013) sought treatment at four different CAP clinics in southern Sweden, in the study. The clinics were the only provider of specialist level care and were situated in average level socio-economic areas for Sweden. Exclusion criterion was the need of interpretation for parents or patient. Forty cases were discarded due to protocol violations (one clinician used leading questions or did not ask both parent and child questions about all symptom areas) or failure of the diagnostician to report data. Another 28 cases were withdrawn due to insufficient additional information in the medical records up until 6 months from intake, a pre-requisite for an adequate LEAD procedure. Data from the remaining $n=239$ cases are reported. Mean age of the 239 participants was 12.1 (sd. 3.1, range 6.1-17.8) years old. The observation time that had yielded new information was 1.2 (sd. 0.6) years with a range 0.1-3.1 years. The proportion of children 6-12 years was $\mathrm{n}=138(57,7 \%)$. There were slightly more boys $(\mathrm{n}=131$, $54.8 \%)$ than girls included and boys were slightly younger than girls (11.9 vs. 12.4 years, $\mathrm{p}=.007)$. Almost all children had clinically significant disorders, with current Children's Global Assessment Scale scores (CGAS) for boys just below $50(\mathrm{~m}=48.0, \mathrm{SD}=0.46)$ and girls $50(\mathrm{~m}=50.0$, $\mathrm{SD}=0.56)$. No one had CGAS scores in the non-clinical range of 70 or above.

Further, out of the 239 patients, a SCARED self-rating scale was filled in by 204 patients (boys $n=112$ /girls $n=92$ respectively) and the corresponding parent-rating scale was filled in by 228 parents (boys $n=122 /$ girls $n=106$ ). Hence, numbers will vary somewhat across analyses.

\section{Measures and Procedures}

A comprehensive description of measures and procedures can be found in a previous report on the KSADS by Jarbin [30]. Shortly, the semi-structured interview, the the Schedule for Affective Disorders and Schizophrenia for School-Age Children- Present and Lifetime Version (K-SADS-PL) was used by resident MDs (either CAP specialists or in training to become CAP specialists) following a training program (see [30]). The KSADS interviews with both parents and patients yielded DSM IV diagnoses which were then further evaluated using a "Longitudinal Expert All Data" (LEAD) process commonly viewed as the best proxy to a "gold standard" that can be used to evaluate semi-structured interviews. Through the LEAD process, all information brought in through diagnostic procedures, the level of impairment, and the outcome of treatment across a suitable time period is used [31-33] for the final LEAD diagnosis or diagnoses (see [31] for a detailed description). To be eligible for LEAD, the record should cover at least 6 months of follow-up from the K-SADS-PL and include at least three further visits or significant information from a teacher or an assessment of a senior clinician. In the LEAD procedure, the judge has access to the K-SADS-PL interview as well as subsequent information from the medical records. All these data were retrieved using a structured form. Thus, the re-evaluation of the KSADS diagnoses was systematic and could include oral reports and report forms from teachers and other informants, psychological assessments and the outcome of pharmacological and psychological treatment. For further information about the reliability etc. of this process, see Jarbin [30]. Furthermore, a research assistant asked the patient and parent to fill in the SCARED (as well as some other scales) during one visit.

\section{Statistics}

T-tests or $\chi^{2}$ tests were conducted to examine gender differences, differences between diagnoses etc. Receiver operating characteristics (ROC) analyses were conducted to examine the concurrent validity of the SCARED total score and subscales [34]. Generally, the area under the curve (AUC) is judged to represent low accuracy between 0.50 and 0.70 , moderate accuracy between 0.70 and 0.90 and $>0.90$ high accuracy [35]. Agreement between LEAD diagnoses and cut-off for the SCARED scales and subscales were studied using the Kappa statistic. Regarding Kappa values a common interpretation is that: Poor agreement $=$ Less than 0.20; Fair agreement $=0.20-0.40$; Moderate agreement $=0.40-0.60$; Good agreement $=0.60-0.80$; Very good agreement $=0.80-1.00$. We also conducted series of logistic regression analysis: first, to evaluate the concurrent and discriminant validity of the SCARED subscales vis-à-vis all anxiety diagnoses; second, sequential logistic regression analyses were conducted to examine whether adding an informant (child or parent) would increase how accurately children with a specific anxiety disorder could be identified based on the relevant SCARED subscale.

\section{Results}

\section{Sample Characteristics}

Based on the LEAD procedure, patients displayed a psychiatric disorder pattern that is representative of that seen in 
Table 1 The frequency of psychiatric disorders in the outpatient sample

\begin{tabular}{|c|c|c|c|c|c|c|}
\hline \multirow[t]{2}{*}{ Psychiatric disorders } & \multicolumn{2}{|c|}{ Boys } & \multicolumn{2}{|c|}{ Girls } & \multicolumn{2}{|l|}{ Total } \\
\hline & $\mathrm{N}$ & $\%$ & $\mathrm{~N}$ & $\%$ & $\mathrm{~N}$ & $\%$ \\
\hline Any affective disorder & 40 & 26.7 & 40 & 34.2 & $80^{\mathrm{a}}$ & 30.0 \\
\hline Anxiety disorder total & 48 & 32.0 & 50 & 42.7 & $98^{\mathrm{a}}$ & 36.7 \\
\hline Separation anxiety disorder (SAD) & 12 & 8.0 & 10 & 8.5 & $22^{\mathrm{a}}$ & 8.2 \\
\hline Social phobia $(\mathrm{SoP})$ & 6 & 4.0 & 13 & 11.1 & $19^{c}$ & 7.1 \\
\hline Generalized anxiety disorder (GAD) & 6 & 4.0 & 10 & 8.5 & $16^{\mathrm{a}}$ & 6.0 \\
\hline Anxiety NOS & 7 & 4.7 & 6 & 5.1 & $13^{\mathrm{a}}$ & 4.9 \\
\hline Obsessive-compulsive disorder & 7 & 4.7 & 5 & 4.3 & $12^{\mathrm{a}}$ & 4.5 \\
\hline Specific phobia (SP) & 26 & 17.3 & 17 & 14.5 & $43^{\mathrm{a}}$ & 16.1 \\
\hline Panic disorder (PD)/Agoraphobia & 2 & 1.3 & 6 & 5.1 & $8^{\mathrm{a}}$ & 3.0 \\
\hline Posttraumatic stress disorder & 0 & 0 & 2 & 1.1 & $2^{\mathrm{a}}$ & 0.7 \\
\hline Any neurodevelopmental disorder ${ }^{\mathrm{d}}$ & 117 & 78 & 68 & 58.1 & $185^{\mathrm{b}}$ & 69.3 \\
\hline Any disruptive behavioural disorder ${ }^{\mathrm{e}}$ & 57 & 38 & 36 & 30.8 & $93^{\mathrm{a}}$ & $34.8 \%$ \\
\hline
\end{tabular}

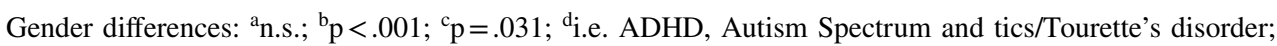
i.e. oppositional-defiant disorder and conduct disorder
CAP-clinics (Table 1) with almost 1/3rd affective disorders, a good $1 / 3$ rd anxiety disorders and many with neuropsychiatric disorders (ADHD, tics/Tourette and Autism Spectrum Disorders) $(69.3 \%)$. In the latter case gender differences were strongly evident for the most prevalent disorder [i.e. ADHD combined type $36.7 \%$ boys $/ 17.9 \%$ girls $\chi^{2}(2,12.341$ $\mathrm{p}=.002)]$, more so than in depressive and anxiety disorders (apart from social phobia), where gender differences were quite small. As the number of patients that had specific anxiety disorders differed, some tests are at risk for type
II errors. However, for most disorders, our study provides enough patients to study psychometric properties of the SCARED-R with sub-scales (Table 1).

\section{Gender and Informant Differences}

Girls scored higher than boys on the SCARED/SCARED$\mathrm{R}$ total scores self-ratings. Such gender differences were less and non-significant in parent ratings, in line with the small gender difference in the prevalence of "any anxiety

Table 2 Means, standard deviations and independent t-test as per diagnostic group for self- respectively parent ratings separately for boys and girls

\begin{tabular}{|c|c|c|c|c|c|c|c|c|}
\hline \multirow[b]{2}{*}{$\begin{array}{l}\text { Scale/subscale (number of } \\
\text { items) }\end{array}$} & \multicolumn{4}{|l|}{ Child rating } & \multicolumn{4}{|l|}{ Parent rating } \\
\hline & $\begin{array}{l}\text { All } \\
\mathrm{N}=204 \\
\mathrm{M}(\mathrm{SD})\end{array}$ & $\begin{array}{l}\text { Boys } \\
\mathrm{N}=112 \\
\mathrm{M}(\mathrm{SD})\end{array}$ & $\begin{array}{l}\text { Girls } \\
\mathrm{n}=92 \\
\mathrm{M}(\mathrm{SD})\end{array}$ & t-test & $\begin{array}{l}\text { All } \\
\mathrm{N}=228 \\
\mathrm{M}(\mathrm{SD})\end{array}$ & $\begin{array}{l}\text { Boys } \\
\mathrm{n}=122 \\
\mathrm{M}(\mathrm{SD})\end{array}$ & $\begin{array}{l}\text { Girls } \\
\mathrm{n}=106 \\
\mathrm{M}(\mathrm{SD})\end{array}$ & t-test \\
\hline $\begin{array}{l}\text { SCARED total score (41 } \\
\text { items) }\end{array}$ & $21.33(12.73)$ & 17.67 (11.99) & $25.79(12.22)^{\mathrm{c}}$ & $-4.774 * * *$ & $16.09(12.42)$ & $15.46(12.30)$ & $16.83(12.57)^{\mathrm{c}}$ & -0.836 \\
\hline $\begin{array}{l}\text { SCARED-R total score ( } 69 \\
\text { items) }\end{array}$ & $35.16(19.89)$ & $29,24(18,89)^{\mathrm{a}}$ & $42.37(18.75)^{\mathrm{c}}$ & $-4.956 * * *$ & $23.19(16.72)$ & $22.21(16,65)^{\mathrm{a}}$ & $24.34(16.81)^{\mathrm{c}}$ & -0.966 \\
\hline SAD (8) & $3.60(2.95)$ & $2.88(2.68)$ & $4.49(3.03)^{\mathrm{c}}$ & $-4.034 * * *$ & $2.94(3,06)$ & $2.71(2.90)$ & $3.21(3.23)^{\mathrm{c}}$ & -1.227 \\
\hline SoP (7) & $5.10(3.36)$ & $4.61(3.36)^{\mathrm{c}}$ & $5.71(3.27)^{\mathrm{c}}$ & $-2.352 *$ & $3,77(4,03)$ & $3.77(4.01)^{\mathrm{c}}$ & $3.77(4.08)^{\mathrm{c}}$ & 0.003 \\
\hline GAD (9) & $5.51(4.23)$ & $4.29(3.91)$ & $7.00(4.16)^{\mathrm{b}}$ & $-4.781 * * *$ & $4.79(4.15)$ & $4.38(4.05)$ & $5.26(4.24)^{\mathrm{b}}$ & -1.611 \\
\hline SchoP (5) & $2.29(2.14)$ & $1.93(1.93)$ & $2.74(2.30)$ & $-2.737 * *$ & $2.30(2.29)$ & $2,30(2,36)$ & $2.30(2.21)$ & 0.016 \\
\hline OCD (9) & $5.21(3.45)$ & $4.83(3.37)^{\mathrm{a}}$ & $5.67(3.51)^{\mathrm{c}}$ & -1.745 & $2.32(2.49)$ & $2,41(2,51)^{\mathrm{a}}$ & $2.22(2.48)^{\mathrm{c}}$ & 0.587 \\
\hline SP (13) & $6.64(4.78)$ & $5.22(4.13)$ & $8.37(4.97)^{\mathrm{c}}$ & $-4.851 * * *$ & $3.99(3.74)$ & $3.61(3.62)$ & $4.43(3.85)^{\mathrm{c}}$ & -1.674 \\
\hline PD (13) & $4.82(4.49)$ & $3.96(4.13)$ & $5.86(4.71)^{\mathrm{c}}$ & $-3.061 * *$ & $2.30(3.84)$ & $2.30(3.86)$ & $2.30(3,84)^{\mathrm{c}}$ & -0.006 \\
\hline APTSD (4) & $1.98(2.24)$ & $1.52(2.06)$ & $2.53(2.33)^{\mathrm{c}}$ & $-3.300 * * *$ & $0.79(1.42)$ & $0.73(1.36)$ & $0.86(1.49)^{\mathrm{c}}$ & -0.703 \\
\hline
\end{tabular}

SCARED subscales: $S A D$ Separation Anxiety Disorder, SoP Social phobia, GAD Generalized anxiety disorder, SchoP School phobia, $O C D$ Obsessive-compulsive disorder, $S P$ Specific phobia, $P D$ Panic disorder, APTSD Acute or post-traumatic stress disorder

$* \mathrm{p}<.05 ; * * \mathrm{p}<.01 ; * * \mathrm{p}<.001$

Significant differences between child and parent report: $\mathrm{a}=\mathrm{p}<.001 ; \mathrm{b}=\mathrm{p}<.01 ; \mathrm{c}=\mathrm{p}<.05$ 
disorder" (Table 2). This pattern, is evident as well at the level of specific anxiety diagnoses except OCD, where selfand parental ratings were comparable.

Thus, the moderate parent-child agreement (range about $r=0.5$ for school phobia and panic disorder to $r=0.37$ for GAD, all $\mathrm{p}<.001$ ) and the particularly low agreement we saw on the OCD-scale $(r=0.21, p=0.004)$, may have different causes. It may be caused by girls' elevated selfratings but also by boys' low self-ratings when compared with parental ratings (Table 2). However, boys' self-ratings varied, and were similar to parents' ratings for some subscales, and even significantly higher than their parents' ratings on two sub-scales (OCD and SoP) (Table 2).

\section{Screening Efficiency}

First, we conducted a series of ROC analyses to evaluate how efficiently the total scores and subscales would predict the presence of any anxiety disorder and specific ADs (See Table 3). All predictions were significant. The childreported total scores (SCARED and SCARED-R) predicted the presence of any AD with low accuracy and the parentreported total scores predicted the presence of any AD with moderate accuracy. The disorder-specific subscales predicted their corresponding disorder with low to moderate accuracy (Table 3).

Secondly, we selected the most efficient cut-offs to minimize false-positive and false-negative equally by focusing on maximizing efficiency $\kappa(0.5)$ [36], and evaluated the sensitivity and specificity of these cut-off scores (Table 3 ).
In all instances, the Kappa $[\kappa(0.5)]$ showed fair agreement between the total scores/subscales and their corresponding diagnosis except that the cut-off values for the GAD subscale had poor agreement with the LEAD GAD diagnosis (Table 3).

Most SCARED/SCARED-R sub-scales for self-ratings showed adequate "Area Under the Curve" (AUC) in ROC analysis (Table 3), ranging from low accuracy for specific phobias ( $\mathrm{AUC}=0.61, \mathrm{p}=0043$ ) to a moderate, bordering on high, $(\mathrm{AUC}=0.89, \mathrm{p}<.001)$ for parental rating of social anxiety OCD (Table 3 ). Thus, most cut-off scores that are based on the ROC analyses (based on the point where both sensitivity and specificity are optimal) can be used with confidence, given that the purpose of its use is in line with the way we estimated it. The sensitivity ranges from the lowest $(60 \%)$ for OCD to the highest (92\%) for PD. The corresponding specificity ranged from the lowest $(60 \%)$ for GAD to the highest (91\%) for OCD.

The SCARED and SCARED-R total scores show corresponding adequate AUC levels, with cut-off yielding high sensitivity, low specificity but still fair kappa levels (Table 3).

Thirdly, we also conducted a series of logistic regression analyses to evaluate the concurrent and discriminant validity of each subscale, i.e. verifying whether only the corresponding subscale of the SCARED would be associated with particular LEAD diagnoses (Table 4). The Odds Ratios (OR), based on logistic regression, shows whether a SCARED/SCARED-R sub-scale relates to the LEAD anxiety diagnoses. It shows, for example that for every

Table 3 Psychometric properties for the SCARED-R versus a LEAD diagnosis of any anxiety disorder and the specific anxiety disorders

\begin{tabular}{|c|c|c|c|c|c|c|c|}
\hline SCARED scale/subscale -> LEAD & Report & AUC & $\mathrm{P}$ & Cut-off & Sensitivity $\%$ & Specificity $\%$ & Kappa \\
\hline \multirow[t]{2}{*}{ SCARED total score $\rightarrow$ Any anxiety } & Child & 0.66 & $<0.001$ & $\geq 15$ & 84 & 43 & 0.23 \\
\hline & Parent & 0.72 & $<0.001$ & $\geq 14$ & 68 & 61 & 0.27 \\
\hline \multirow[t]{2}{*}{ SCARED-R total score $\rightarrow$ Any anxiety } & Child & 0.65 & $<0.001$ & $\geq 25$ & 84 & 43 & 0.23 \\
\hline & Parent & 0.72 & $<0.001$ & $\geq 34$ & 43 & 89 & 0.34 \\
\hline \multirow[t]{2}{*}{$\mathrm{SAD} \rightarrow \mathrm{SAD}$} & Child & 0.76 & $<0.001$ & $\geq 5$ & 78 & 70 & 0.21 \\
\hline & Parent & 0.84 & $<0.001$ & $\geq 5$ & 79 & 80 & 0.32 \\
\hline \multirow[t]{2}{*}{$\mathrm{SoP} \rightarrow \mathrm{SoP}$} & Child & 0.85 & $<0.001$ & $\geq 8$ & 77 & 80 & 0.29 \\
\hline & Parent & 0.89 & $<0.001$ & $\geq 9$ & 75 & 88 & 0.39 \\
\hline \multirow[t]{2}{*}{$\mathrm{GAD} \rightarrow \mathrm{GAD}$} & Child & 0.71 & 0.008 & $\geq 6$ & 79 & 60 & 0.11 \\
\hline & Parent & 0.74 & 0.002 & $\geq 8$ & 56 & 80 & 0.17 \\
\hline \multirow[t]{2}{*}{$\mathrm{OCD} \rightarrow \mathrm{OCD}$} & Child & 0.84 & $<0.001$ & $\geq 10$ & 60 & 91 & 0.31 \\
\hline & Parent & 0.84 & $<0.001$ & $\geq 9$ & 46 & 97 & 0.42 \\
\hline \multirow[t]{2}{*}{$\mathrm{SP} \rightarrow \mathrm{SP}$} & Child & 0.61 & 0.043 & $\geq 7$ & 68 & 57 & 0.13 \\
\hline & Parent & 0.68 & 0.001 & $\geq 5$ & 63 & 70 & 0.21 \\
\hline \multirow[t]{2}{*}{$\mathrm{PD} \rightarrow \mathrm{PD}$} & Child & 0.84 & $<0.001$ & $\geq 6$ & 92 & 69 & 0.18 \\
\hline & Parent & 0.88 & $<0.001$ & $\geq 4$ & 83 & 83 & 0.28 \\
\hline
\end{tabular}

$A U C$ Area under the curve, $S A D$ separation anxiety disorder, $S P$ specific phobia, $S o P$ social phobia, $G A D$ generalized anxiety disorder, $P D$ panic disorder, $O C D$ obsessive-compulsive disorder, $A P T S D$ acute or post-traumatic stress disorder 
Table 4 Convergent/divergent validity of the SCARED child- and parent report versus LEAD diagnoses using logistic regression where the Odds Ratio (OR) refers to the likelihood of a diagnosis for every additional score point on the scale/sub-scale

\begin{tabular}{|c|c|c|c|c|c|c|}
\hline \multirow[t]{2}{*}{ SCARED-R } & \multicolumn{6}{|l|}{ LEAD diagnoses } \\
\hline & $\begin{array}{l}\text { SAD OR }(95 \% \\
\text { CI) P }\end{array}$ & SP OR $(95 \%$ CI) P & SoP OR (95\% CI) P & PD OR $(95 \%$ CI $)$ P & $\begin{array}{l}\text { GAD OR }(95 \% \\
\text { CI) P }\end{array}$ & OCD OR $(95 \% \mathrm{CI}) \mathrm{P}$ \\
\hline \multicolumn{7}{|l|}{ Child-report } \\
\hline$\chi^{2}, \mathrm{P}$ & $20.066, p=.003$ & $5.53 \mathrm{p}=.478$ & $33.067, \mathrm{p}<.001$ & $13.155, p=.041$ & $19.271, p=.004$ & $16.065, p=.013$ \\
\hline SAD & $\begin{array}{l}1.50(1.21 \\
1.85)<0.001\end{array}$ & $\begin{array}{l}0.93(0.78,1.11) \\
0.440\end{array}$ & $\begin{array}{l}0.96(0.76,1.22) \\
0.746\end{array}$ & $\begin{array}{l}1.07(0.76,1.50) \\
0.690\end{array}$ & $\begin{array}{l}1.23(0.98,1.54) \\
0.078\end{array}$ & $\begin{array}{l}0.95(0.71,1.26) \\
0.716\end{array}$ \\
\hline SP & $\begin{array}{l}0.92(0.80,1.05) \\
0.228\end{array}$ & $\begin{array}{l}1.10(0.99,1.23) \\
0.075\end{array}$ & $\begin{array}{l}0.93(0.79,1.09) \\
0.347\end{array}$ & $\begin{array}{l}0.97(0.78,1.19) \\
0.753\end{array}$ & $\begin{array}{l}0.96(0.82,1.11) \\
0.551\end{array}$ & $\begin{array}{l}1.09(0.91,1.30) \\
0.336\end{array}$ \\
\hline SoP & $\begin{array}{l}1.07(0.90,1.28) \\
0.439\end{array}$ & $\begin{array}{l}0.92(0.81,1.04) \\
0.190\end{array}$ & $\begin{array}{l}1.55(1.25 \\
1.94)<0.001\end{array}$ & $\begin{array}{l}1.05(0.81,1.35) \\
0.731\end{array}$ & $\begin{array}{l}1.17(0.96,1.43) \\
0.111\end{array}$ & $\begin{array}{l}0.98(0.79,1.21) \\
0.844\end{array}$ \\
\hline PD & $\begin{array}{l}1.01(0.85,1.20) \\
0.905\end{array}$ & $\begin{array}{l}1.03(0.91,1.17) \\
0.650\end{array}$ & $\begin{array}{l}1.12(0.95,1.33) \\
0.187\end{array}$ & $\begin{array}{l}1.18(0.95,1.48) \\
0.142\end{array}$ & $\begin{array}{l}1.02(0.86,1.21) \\
0.835\end{array}$ & $\begin{array}{l}0.93(0.76,1.14) \\
0.486\end{array}$ \\
\hline GAD & $\begin{array}{l}0.88(0.72,1.06) \\
0.177\end{array}$ & $\begin{array}{l}0.96(0.84,1.11) \\
0.596\end{array}$ & $\begin{array}{l}1.09(0.92,1.29) \\
0.314\end{array}$ & $\begin{array}{l}1.16(0.89,1.51) \\
0.265\end{array}$ & $\begin{array}{l}1.23(1.02,1.49) \\
0.030\end{array}$ & $\begin{array}{l}1.05(0.84,1.33) \\
0.655\end{array}$ \\
\hline OCD & $\begin{array}{l}1.00(0.81,1.23) \\
0.996\end{array}$ & $\begin{array}{l}1.04(0.89,1.21) \\
0.633\end{array}$ & $\begin{array}{l}0.83(0.66,1.05) \\
0.114\end{array}$ & $\begin{array}{l}0.91(0.66,1.26) \\
0.582\end{array}$ & $\begin{array}{l}0.84(0.66,1.08) \\
0.170\end{array}$ & $\begin{array}{l}1.39(1.08,1.78) \\
0.009\end{array}$ \\
\hline \multicolumn{7}{|l|}{ Parent-report } \\
\hline$\chi^{2}, \mathrm{P}$ & $42.811, \mathrm{p}<.001$ & $11.268, p=.080$ & $43.997,<0.001$ & $19.027, p=.004$ & $16.549, p=.011$ & $24.060, p=.001$ \\
\hline SAD & $\begin{array}{l}1.79(1.40 \\
2.28)<0.001\end{array}$ & $\begin{array}{l}0.96(0.84,1.11) \\
0.610\end{array}$ & $\begin{array}{l}0.78(0.61,1.00) \\
0.054\end{array}$ & $\begin{array}{l}1.06(0.79,1.43) \\
0.688\end{array}$ & $\begin{array}{l}1.01(0.83,1.22) \\
0.959\end{array}$ & $\begin{array}{l}0.86(0.66,1.11) \\
0.237\end{array}$ \\
\hline SP & $\begin{array}{l}0.78(0.62,097) \\
0.027^{*}\end{array}$ & $\begin{array}{l}1.13(1.01,1.26) \\
0.027\end{array}$ & $\begin{array}{l}1.11(0.94,1.32) \\
0.224\end{array}$ & $\begin{array}{l}1.00(0.81,1.24) \\
0.987\end{array}$ & $\begin{array}{l}1.06(0.90,1.24) \\
0.497\end{array}$ & $\begin{array}{l}1.14(0.96,1.35) \\
0.134\end{array}$ \\
\hline SoP & $\begin{array}{l}1.11(0.96,1.29) \\
0.172\end{array}$ & $\begin{array}{l}1.09(0.99,1.19) \\
0.097\end{array}$ & $\begin{array}{l}1.47(1.23 \\
1.77)<0.001\end{array}$ & $\begin{array}{l}0.89(0.71,1.13) \\
0.340\end{array}$ & $\begin{array}{l}1.12(0.98,1.29) \\
0.092\end{array}$ & $\begin{array}{l}0.89(0.74,1.08) \\
0.247\end{array}$ \\
\hline PD & $\begin{array}{l}1.14(0.94,1.37) \\
0.176\end{array}$ & $\begin{array}{l}0.99(0.88,1.11) \\
0.874\end{array}$ & $\begin{array}{l}0.96(0.83,1.10) \\
0.530\end{array}$ & $\begin{array}{l}1.15(0.98,1.35) \\
0.086\end{array}$ & $\begin{array}{l}1.03(0.90,1.17) \\
0.667\end{array}$ & $\begin{array}{l}1.09(0.93,1.28) \\
0.266\end{array}$ \\
\hline GAD & $\begin{array}{l}0.78(0.62,0.97) \\
0.28^{*}\end{array}$ & $\begin{array}{l}1.01(0.90,1.12) \\
0.919\end{array}$ & $\begin{array}{l}1.21(1.03,1.43) \\
0.022\end{array}$ & $\begin{array}{l}1.32(1.02,1.71) \\
0.038\end{array}$ & $\begin{array}{l}1.18(1.01,1.37) \\
0.035\end{array}$ & $\begin{array}{l}1.02(0.84,1.23) \\
0.861\end{array}$ \\
\hline OCD & $\begin{array}{l}0.90(0.65,1.26) \\
0.544\end{array}$ & $\begin{array}{l}1.00(0.83,1.21) \\
0.975\end{array}$ & $\begin{array}{l}0.97(0.75,1.24) \\
0.776\end{array}$ & $\begin{array}{l}1.05(0.77,1.43) \\
0.767\end{array}$ & $\begin{array}{l}0.88(0.68,1.13) \\
0.308\end{array}$ & $\begin{array}{l}1.47(1.12,1.94) \\
0.006\end{array}$ \\
\hline
\end{tabular}

*Low SP score $=$ more likelihood of LEAD SAD

additional point scored on the child SAD scale or the parent SAD scale, the likelihood increases $1.42(\mathrm{p}=.001)$, respectively $1.59(\mathrm{p}=.001)$ times, that a SAD diagnosis will be present. Likewise, the other SCARED scales (e.g. SoP and GAD) were significantly respectively tended to be related to their respective LEAD diagnoses, but the child SoP scale tended to predict a GAD as well but the GAD sub-scale did not predict SoP (see Table 4 for figures). On the other hand, the parent SoP sub-scale discriminated well against both SAD and GAD, but the parental GAD scale increased the likelihood of both a GAD $(\mathrm{OR}=1,14, \mathrm{p}=.055)$, a SoP $(\mathrm{OR}=1,19, \mathrm{p}=.026)$ and a PD diagnosis $(\mathrm{OR}=1,34$, $\mathrm{p}=.018$ ) but decreased the likelihood of a SAD diagnosis $(\mathrm{OR}=.79, \mathrm{p}=.013)$.

For the additional SCARED-R scales, the OCD scales worked best $(\mathrm{OR}=1.39, \mathrm{p}=0$ 009) and $(\mathrm{OR}=1.47$, $\mathrm{p}=.006)$ respectively with limited overlap against other diagnoses. However, both the SP and PD scales were weaker predictors of their "own" diagnosis and related to one other diagnosis each OCD and SAD respectively.

\section{Adding Parent Information to Child Information (and Vice Versa)}

We also evaluated the possible benefit of adding the SCARED parent-report to the child-report (and vice versa) in predicting the presence of LEAD ADs, using a sequential logistic regression analysis, to evaluate whether the SCARED total score and subscales would predict the presence/absence of any anxiety disorder (SAD, SoP, GAD, OCD, SP and, PD). We entered the child-report first and then added the parent-report following which we started out with the parent-report first and then added the childreport. Thus, the unique contribution of each informant to the other was evaluated. 
Table 5 Sequential logistic regression to test the effects of child and parent report on the SCARED for the prediction of any anxiety disorder or specific anxiety disorders

\begin{tabular}{|c|c|c|c|c|c|c|}
\hline LEAD diagnosis & $\begin{array}{l}\text { SCARED } \\
\text { Scale_-Informant }\end{array}$ & OR $(95 \%)$ & Wald & Full model $\chi^{2}$ & $\begin{array}{l}\text { Full model adding } \\
\text { an extra report } \chi^{2} \text { a }\end{array}$ & $\mathrm{R}^{2}$ \\
\hline \multirow[t]{2}{*}{ Any anxiety univariate models } & Revised total—child & $3.98(1.95,8.11)$ & $14.389 * * *$ & $16.543 * * *$ & & 0.11 \\
\hline & Revised total-parent & $5.79(2.82,11.91)$ & $22.844 * * *$ & $25.116^{* * *}$ & & 0.16 \\
\hline \multirow[t]{2}{*}{ Any anxiety multivariate model } & Revised total—child & $3.23(1.54,6.79)$ & $9.551 * *$ & $35.624 * * *$ & $\Delta 10.508 * * *$ & 0.22 \\
\hline & Revised total-parent & $4.89(2.33,10.25)$ & $17.687 * * *$ & $35.624 * * *$ & $\Delta 19.081 * * *$ & \\
\hline \multirow{2}{*}{$\begin{array}{l}\text { Any anxiety } \\
\text { univariate model }\end{array}$} & Total score—child & $3.85(1.88,7.85)$ & $13.687 * * *$ & $15.678 * * *$ & & 0.10 \\
\hline & Total score-parent & $2.92(1.60,5.32)$ & $12.255^{* * *}$ & $12.801 * * *$ & & 0.09 \\
\hline \multirow{2}{*}{$\begin{array}{l}\text { Any anxiety } \\
\text { Multivariate model }\end{array}$} & Total score—child & $3.26(1.57,6.77)$ & $10.048 * *$ & $23.890 * * *$ & $\Delta 11.089 * * *$ & 0.16 \\
\hline & Total score-parent & $2.45(1.32,4.56)$ & $8.030 * *$ & $23.890 * * *$ & $\Delta 8.212 * *$ & \\
\hline \multirow[t]{2}{*}{ SAD univariate models } & SAD - child & $7.96(2.51,25.26)$ & $12.371 * * *$ & $15.367 * * *$ & & 0.16 \\
\hline & SAD_parent & $15.59(4.82,50.41)$ & $21.042 * * *$ & $26.444 * * *$ & & 0.27 \\
\hline \multirow{2}{*}{$\begin{array}{l}\text { SAD } \\
\text { Multivariate model }\end{array}$} & SAD—child & $3.18(0.88,11.54)$ & 3.096 & $29.774 * * *$ & $\Delta 3.330$ & 0.31 \\
\hline & SAD_parent & $9.49(2.67,33.82)$ & $12.057 * * *$ & $29.774 * * *$ & $\Delta 14.407 * * *$ & \\
\hline \multirow[t]{2}{*}{ SoP univariate models } & SoP—child & $11.76(3.59,38.56)$ & $16.537 * * *$ & $19.787 * * *$ & & 0.22 \\
\hline & SoP-parent & $20.74(6.17,69.77)$ & $23.997 * * *$ & $28.608 * * *$ & & 0.31 \\
\hline \multirow{2}{*}{$\begin{array}{l}\text { SoP } \\
\text { Multivariate model }\end{array}$} & SoP_child & $6.29(1.74,22.75)$ & $7.868 * *$ & $37.232 * * *$ & $\Delta 8.624 * *$ & 0.40 \\
\hline & SoP-parent & $12.72(3.56,45.44)$ & $15.314 * * *$ & $37.232 * * *$ & $\Delta 17.445^{* * *}$ & \\
\hline \multirow{2}{*}{$\begin{array}{l}\text { GAD } \\
\text { Univariate models }\end{array}$} & GAD—child & $5.45(1.47,20.20)$ & $6.435^{*}$ & $7.976 * *$ & & 0.10 \\
\hline & GAD—parent & $6.29(1.99,19.77)$ & $9.854 * *$ & $10.318 * * *$ & & 0.13 \\
\hline \multirow{2}{*}{$\begin{array}{l}\text { GAD } \\
\text { Multivariate model }\end{array}$} & GAD—child & $3.98(1.04,15.32)$ & $4.039 *$ & $15.043 * * *$ & $\Delta 4.725^{*}$ & 0.18 \\
\hline & GAD_parent & $4.77(1.47,15.49)$ & $6.759 * *$ & $15.043 * * *$ & $\Delta 7.067 * *$ & \\
\hline \multirow{2}{*}{$\begin{array}{l}\text { OCD } \\
\text { Univariate models }\end{array}$} & OCD—child & $14.17(3.65,54.93)$ & $14.698 * * *$ & $14.118 * * *$ & & 0.21 \\
\hline & OCD_parent & $36.60(7.96,168.20)$ & $21.406 * * *$ & $19.201 * * *$ & & 0.28 \\
\hline \multirow{2}{*}{$\begin{array}{l}\text { OCD } \\
\text { Multivariate model }\end{array}$} & OCD—child & $18.18(3.21,102.83)$ & $10.758 * * *$ & $31.140 * * *$ & $\Delta 11.938 * * *$ & 0.44 \\
\hline & OCD—parent & $47.74(7.07,322.47)$ & $15.734 * * *$ & $31.140 * * *$ & $\Delta 17.021 * * *$ & \\
\hline \multirow{2}{*}{$\begin{array}{l}\text { SP } \\
\text { Univariate models }\end{array}$} & SP—child & $3.04(1.31,7.02)$ & $6.743 * *$ & $7.321 * *$ & & 0.06 \\
\hline & $\mathrm{SP}$-parent & $3.96(1.76,8.93)$ & $11.042 * * *$ & $11.558 * * *$ & & 0.10 \\
\hline \multirow{2}{*}{$\begin{array}{l}\text { SP } \\
\text { Multivariate model }\end{array}$} & $\mathrm{SP}$ —child & $2.17(0.90,5.26)$ & 2.963 & $14.640 * * *$ & $\Delta 3.082$ & 0.12 \\
\hline & $\mathrm{SP}$-parent & $3.17(1.36,7.39)$ & $7.085^{* *}$ & $14.640 * * *$ & $\Delta 7.319^{* *}$ & \\
\hline
\end{tabular}

In the multivariate model, the contribution of the child to the parent report and vice versa are added to the values in the single report from the uni-variate analysis (e.g., parent $\mathrm{OR}=4.89$ are added to the child $\mathrm{OR}=3.89$ ). The $\Delta 19.081$ is the difference between the full model $\chi^{2}$ $35.624-16.543$ etc

The final results of the logistic regression analyses are shown in Table 5. The goodness-of-fit in testing the prediction of anxiety disorders was good in all cases (Hosmer-Lemeshow $\mathrm{p}>.05$ ). In single variable models, both the child- and parent-reports of the SCARED-R total score predicted the presence of any anxiety disorder, and ORs were $(3.98,95 \%$ CI $1.95,8.11$ and 5.79, 95\% CI 2.82, 11.91 respectively) explaining $\left(R^{2}\right) 0.11$ and 0.16 respectively proportion of the variation. However, in both instances we observed significant benefits of adding the parent-report to the child report $\left(\Delta \chi_{\text {Parent }}^{2}=19.081\right.$, $\mathrm{p}<.001)$ respectively adding the child-report to the parent-report $\left(\Delta \chi^{2}\right.$ Child $\left.=10.508, \mathrm{p}<.001\right)$. In the final model both child- and parent-report each contributed significantly to the prediction of any anxiety disorder (see Table 5 for OR and $\chi^{2}$ ).
For the SCARED total score both child-, and parentreport predicted the presence of any anxiety disorder in a single-variable model (OR were 3.85, 95\% CI 1.88, 7.85 and $2.92,95 \%$ CI 1.60, 5.32 respectively) with $\mathrm{R}^{2} 0.10$ and 0.09 respectively. In both instances the model improved by adding the other informant $\left(\Delta \chi_{\text {Parent }}^{2}=8.212, \mathrm{p}<.01\right.$ and $\left.\Delta \chi_{\text {Child }}^{2}=11.089, \mathrm{p}<.001\right)$ (see Table 5 for OR and $\chi^{2}$ ).

For the SAD subscale both versions were significant in the single variable model $(\mathrm{OR}=7.96,95 \%$ CI $2.51,25.26$ and $\mathrm{OR}=15.59,95 \% \mathrm{CI} 4.82,50.41)$ with $\mathrm{R}^{2} 0.16$ and 0.27 respectively. However, the model improved only by adding parent-report to the child-report $\left(\Delta \chi_{\text {Parent }}^{2}=14.407\right.$, $\mathrm{p}<.001)$ but not by adding the child-report to the parentreport $\left(\Delta \chi^{2}\right.$ Child $\left.=3.30\right)$ (see Table 5 for OR and $\chi^{2}$ ).

For the SoP subscales both child- and parent-report showed significant ORs in the single variable model (OR 
were $11.76,95 \%$ CI $3.59,38.56$ and $20.74,95 \%$ CI 6.17 , 69.77 respectively) with $R^{2} 0.22$ and 0.31 respectively. Both models improved by adding an extra informant $\left(\Delta \chi_{\text {Parent }}^{2}=17.445, \mathrm{p}<.001\right.$ and $\left.\Delta \chi_{\text {Child }}^{2}=8.624, \mathrm{p}<.01\right)$ (see Table 5 for OR and $\chi^{2}$ ).

We found statistically significant associations between the GAD subscales and the presence of GAD both for the child-report and the parent report (OR were $5.45,95 \% \mathrm{CI}$ $1.47,20.20$ and $6.29,95 \%$ CI $1.99,19.77$ respectively) with $\mathrm{R}^{2} 0.10$ and 0.13 respectively individually. Both models improved by adding an informant $\left(\Delta \chi_{\text {Parent }}^{2}=7.067, \mathrm{p}<.01\right.$ and $\chi_{\text {Child }}^{2}=4.725, \mathrm{p}<.05$ ) (see Table 5 for OR and $\chi^{2}$ ).

Next, we analysed whether the OCD subscales would predict the presence/absence of a LEAD OCD diagnosis, which both the child-report $(\mathrm{OR}=14.17,95 \%$ CI 3.65 , 54.93, $\left.\mathrm{R}^{2}=0.21\right)$ and parent-report $(\mathrm{OR}=36.60,95 \%$ CI $\left.7.96,168.20, \mathrm{R}^{2}=0.28\right)$ did. In addition, the model improved by adding an extra informant $\left(\Delta \chi^{2}\right.$ Parent $=17.021$, $\mathrm{p}<.001$ and $\Delta \chi^{2}$ Child $=11.938, \mathrm{p}<.001$ ) (see Table 5 for OR and $\chi^{2}$ ).

We also assessed whether the SP subscales would predict the presence or absence of a LEAD SP diagnosis. Individually, both the child-report $(\mathrm{OR}=3.04,95 \% \mathrm{CI}$ $1.31,7.02, \mathrm{R} 2=0.06)$ and the parent-report $(\mathrm{OR}=3.96$, 95\% CI 1.76, 8.93, $\mathrm{R}^{2}=0.10$ ) had significant association with the SP diagnosis. We also observed a significantly improved model by adding the parent-report to the childreport $\left(\Delta \chi_{\text {Parent }}^{2}=7.319, \mathrm{p}<.01\right)$. However, adding the child-report to the parent-report did not improve the overall model $\left(\Delta \chi_{\text {Child }}^{2}=3.083\right.$, n.s.) (see Table 5 for OR and $\left.\chi^{2}\right)$.

\section{Discussion}

The study is the first to evaluate the utility of the SCARED$\mathrm{R}$ scale and sub-scales based on the psychometric properties versus the gold-standard called LEAD [31-33]. It can thus be expected to provide the most accurate assessment of its usefulness and validity of its predictions. The population that we used was child-psychiatric outpatients having a level of co-morbidity that can be expected from a general clinical population (Table 1) [37]. Thus, the assessments include the various confounds that the clinician is confronted with in the clinic.

First, the SCARED original scale (i.e. that published by Birmaher [21]) and which assesses the three "classical" child anxiety disorders (separation anxiety disorder, social anxiety disorder and generalized anxiety disorder), and panic disorder could be shown to be a valid measure with regard to each of these four diagnostic entities (kappa values against LEAD ranged from 0.11 to 0.21 for selfratings and from 0.17 to 0.39 for parental ratings). Moreover, the three classical anxiety disorders and panic disorder
SCARED scales showed moderate AUC values. Generally, the area under the curve (AUC) is judged to represent low accuracy between 0.50 and 0.70 , moderate accuracy between 0.70 and 0.90 and above 0.90 high accuracy [35].

The cut-off values for these scales rendered acceptable sensitivity and specificity. However, there are caveats, mainly based on the limited agreement between the child and the parent (ranging from 0.37 to 0.48 ) and the poor agreement against LEAD for GAD (Table 3). Despite this, adding an informant is worthwhile and increases the diagnostic precision (Table 5).

Although, the gender differences may point to a general bias for under-reporting symptoms in boys and overreporting symptoms in girls, parents' ratings were not so far above boys' self-ratings. Possibly, girls' reports are the bigger problem, and one can speculate whether their scores reflect a different judgment of severity, or if the scale measures "en passant" other phenomena than just anxiety disorders. Despite these caveats, we conclude that the three classical SCARED sub-scales show good psychometric properties in line with previous studies [15] and can be used with confidence using the cut-off values we provide (Table 3).

School phobia is not a DSM IV/5 diagnosis so that its psychometric properties cannot be studied versus an anxiety specific LEAD diagnosis.

The situation with regard to the added subscales in the SCARED-R [27] is similar. Sub-scales for OCD and specific phobia were studied (as A/PTSD had only two cases the psychometric properties are not discussed). These sub-scales were valid measures regarding corresponding two diagnostic entities (kappa values against LEAD were respectively 0.13 and 0.31 for self-ratings and 0.42 and 0.21 for parental ratings). Moreover, these scales showed moderate AUC values, and the cut-off values for these scales rendered acceptable sensitivity and specificity. However, there are caveats, mainly based on the limited agreement between the child and the parent (ranging from 0.12 to 0.47 ).

Moreover, the gender differences may point to a general bias for under-reporting symptoms in boys and over-reporting symptoms in girls. Despite these caveats, we conclude that the two added SCARED sub-scales show acceptable psychometric properties.

However, a clinician must consider for what purpose the scale is used. The cut-off values described for each scale or sub-scale are mathematical constructs rather than, for example a useful cut-off value for screening. Cut-off values in Table 3 are points on the ROC-curves where the most optimal compromise between sensitivity and specificity is obtained. In situations when screening for anxiety disorders in the clinic, it may be proper to use a lower cut-off score with a higher sensitivity with a concomitant lower 
specificity. Thus, the purpose must dictate what cut-off score to use. Our data indicate that the SCARED-R is useful as a screen for the classical anxiety disorders, but also for OCD, panic and specific phobias.

In primary/first line child psychiatric care, the two total scores may have some usefulness, e.g. screening for the level of anxiety without wishing to pinpoint specific diagnoses. In such cases, child ratings with a sensitivity of about $80 \%$ may be preferable.

Despite the caveats described above, adding the parent as an informant, or vice versa is worthwhile and increases the diagnostic precision (Table 5), contrary to one previous report [15]. However, with regard to SCARED SP respectively SCARED SAD only parent reports added to the child report, while the child report did not increase diagnostic precision to the parental report. In all other instances, both the child and the parent report added to the parent respectively child report, thus increasing diagnostic precision.

Moreover, while using the SCARED-R, the clinician should keep in mind that the properties of this scale are related to the DSM IV anxiety disorders constructs [22], and that these properties may differ from those of DSM-5 diagnoses in some ways.

\section{Summary}

Both parents and children provide unique information, contributing in most diagnostic areas to a correct diagnosis. We urge clinicians to use information from both the child and the parent both when using a scale like the SCARED/ SCARED-R and when assessing within an un-structured clinical interview. In most cases, each provides unique information. However, at which items this contribution is most crucial is not known, but could be studied in this sample.

First, even if this is a sizable study, the number of participants in some diagnostic groups were too small, i.e. we had only two patients diagnosed with a post-traumatic stress disorder. Moreover, just eight patients with panic disorder/agoraphobia increases the risk of error. The 12 patients with OCD was as well rather few. However, for the major "classical" anxiety disorders numbers were clearly adequate so that the concurrent and discriminant validity of these measures can be viewed as established.

Secondly, LEAD diagnoses based on enhancing KSADS with systematic diagnosis related information up to 6 months from inclusion, is still at some risk for chance variation in the information that is available within the medical records. As the SCARED was not a base for the LEAD diagnoses, artificially elevated validity measures are excluded.
Thirdly, the data that our study provides needs to be seen within the context of a normative study, for example regarding gender differences. A Norwegian study, i.e. from a culture close to the Swedish culture, of the SCARED showed data that are compatible with our findings [2] and showed gender differences that were quite similar to our findings.

The SCARED/SCARED-R is generally a valid and reliable screening tool. Particularly the classical SCARED has good psychometric properties and can be used with confidence. However, the added sub-scales (i.e., SCARED$\mathrm{R}$ ) shows more disparate findings, The OCD scale shows mixed results regarding validity/screening efficiency, which, despite a limited patient base, was statistically significant. Regarding the SP scale, the psychometrics were not as good, despite an adequate number of patients.

The cut-off scores we provide represent a compromise between sensitivity and specificity, optimizing both. However, if a particular use of the SCARED scale presupposes, for example, a higher sensitivity, so that a lower cut-off score is used, then unavoidably specificity will be lower than the figures we provide, and the statistical association with the diagnoses will be changed. In general, but less exactly, the figures for adding child to parent information and vice versa are applicable.

Funding Grants from Scientific Research Council in Region Halland and Söderström-Königska Foundation, who had no impact on the design or report.

Open Access This article is distributed under the terms of the Creative Commons Attribution 4.0 International License (http:// creativecommons.org/licenses/by/4.0/), which permits unrestricted use, distribution, and reproduction in any medium, provided you give appropriate credit to the original author(s) and the source, provide a link to the Creative Commons license, and indicate if changes were made.

\section{References}

1. Cohen P, Cohen J, Kasen S, Velez CN (1993) An epidemiological study of disorders in late childhood and adolescence: I Age- and gender-specific prevalence. J Child Psychol Psychiatry 34:851-867

2. Leikanger E, Ingul JM, Larsson B (2012) Sex and age-related anxiety in a community sample of Norwegian adolescents. Scand J Psychol 53:150-157

3. Leikanger E, Larsson B (2012) One-year stability, change and incidence in anxiety symptoms among early adolescents in the general population. Eur Child Adolesc Psychiatry 21:493-501

4. Ginsburg GS et al (2014) Naturalistic follow-up of youths treated for pediatric anxiety disorders. JAMA Psychiatry 71:310-318

5. Copeland WE, Angold A, Shanahan L, Costello EJ (2014) Longitudinal patterns of anxiety from childhood to adulthood: the great smoky mountains study. J Am Acad Child Adolesc Psychiatry 53:21-33 
6. Langley AK, Lewin AB, Bergman R, Lee JC, Piacentini J (2010) Correlates of comorbid anxiety and externalizing disorders in childhood obsessive compulsive disorder. Eur Child Adolesc Psychiatry 19:637-645

7. Bodden DH, Dirksen CD, Bogels SM (2008) Societal burden of clinically anxious youth referred for treatment: a cost-of-illness study. J Abnorm Child Psychol 36:487-497

8. Gren-Landell M, Aho N, Carlsson E, Jones A, Svedin CG (2013) Posttraumatic stress symptoms and mental health services utilization in adolescents with social anxiety disorder and experiences of victimization. Eur Child Adolesc Psychiatry $22: 177-184$

9. Hansen BH, Oerbeck B, Skirbekk B, Kristensen H (2015) Nonobsessive-compulsive anxiety disorders in child and adolescent mental health services-Are they underdiagnosed, and how accurate is referral information? Nord J Psychiatry 70:133-139

10. Heiervang E et al (2007) Psychiatric disorders in Norwegian 8to 10-year-olds: an epidemiological survey of prevalence, risk factors, and service use. J Am Acad Child Adolesc Psychiatry 46:438-447

11. Wang PS et al (2005) Failure and delay in initial treatment contact after first onset of mental disorders in the National Comorbidity Survey Replication. Arch Gen Psychiatry 62:6003-6613

12. Heyman I et al (2001) Prevalence of obsessive-compulsive disorder in the British nationwide survey of child mental health. Br J Psychiatry 179:324-329

13. Pereira AI et al (2015) Agreement and discrepancy between mother and child in the evaluation of children's anxiety symptoms and anxiety life interference. Eur Child Adolesc Psychiatry 24:327-337

14. De Los Reyes A, Kazdin AE (2005) Informant discrepancies in the assessment of childhood psychopathology: a critical review, theoretical framework, and recommendations for further study. Psychol Bull 131:483-509

15. Cosi S, Canals J, Hernandez-Martinez C, Vigil-Colet A (2010) Parent-child agreement in SCARED and its relationship to anxiety symptoms. J Anxiety Disord 24:129-133

16. Grills AE, Ollendick TH (2003) Multiple informant agreement and the anxiety disorders interview schedule for parents and children. J Am Acad Child Adolesc Psychiatry 42:30-40

17. Muris P, Meesters C, Schouten E (2002) A brief questionnaire of DSM-IV-defined anxiety and depression symptoms among children. Clin Psychol Psychother 9:430-442

18. Muris $P$ et al (2017) The youth anxiety measure for DSM-5 (YAM-5): development and first psychometric evidence of a new scale for assessing anxiety disorders symptoms of children and adolescents. Child Psychiatry Hum Dev 48:1-17

19. American Psychiatric Association (2013) Diagnostic and statistical manual of mental disorders (DSM-5), 5th edn. American Psychiatric Publishing, Washington DC

20. Simon E, Bos AER, Verboon P, Smeekes S, Muris P (2017) Psychometric properties of the youth anxiety measure for DSM-5 (YAM-5) in a community sample. Personal Individ Differ 116:258-264

21. Birmaher B et al (1997) The screen for child anxiety related emotional disorders (SCARED): scale construction and psychometric characteristics. J Am Acad Child Adolesc Psychiatry 36:545-553

22. American Psychiatric Association (1994) Diagnostic and statistical manual of mental disorders (DSM IV), 4th edn. American Psychiatric Association, Washington DC

23. Birmaher B et al (1999) Psychometric properties of the screen for child anxiety related emotional disorders (SCARED): a replication study. J Am Acad Child Adolesc Psychiatry 38:1230-1236

24. Monga $S$ et al (2000) Screen for child anxiety-related emotional disorders (SCARED): convergent and divergent validity. Depress Anxiety 12:85-91

25. Muris P, Mayer B, Bartelds E, Tierney S, Bogie N (2001) The revised version of the screen for child anxiety related emotional disorders (SCARED-R): treatment sensitivity in an early intervention trial for childhood anxiety disorders. Br J Clin Psychol 40:323-336

26. Muris P, Steerneman P (2001) The revised version of the screen for child anxiety related emotional disorders (SCARED-R): first evidence for its reliability and validity in a clinical sample. $\mathrm{Br} \mathrm{J}$ Clin Psychol 40:35-44

27. Muris P, Dreessen L, Bogels S, Weckx M, van Melick M (2004) A questionnaire for screening a broad range of DSM-defined anxiety disorder symptoms in clinically referred children and adolescents. J Child Psychol Psychiatry 45:813-820

28. Thomsen PH et al (2013) The Nordic long-term OCD treatment study (NordLOTS): rationale, design, and methods. Child Adolesc Psychiatry Ment Health 7:41

29. Jarbin H, Ivarsson T (2013) Validation of the Swedish translation of the Kiddie Schedule før Affective Disorders and Schizophrenia (KSADS) using Longitudinal Expert All Data (LEAD) diagnoses.

30. Jarbin H, Andersson M, Rastam M, Ivarsson T (2017) Predictive validity of the K-SADS-PL 2009 version in school-aged and adolescent outpatients. Nord J Psychiatry 71:270-276

31. Spitzer RL (1983) Psychiatric diagnosis: are clinicians still necessary? Compr Psychiatry 24:399-411

32. Kranzler HR, Tennen H, Babor TF, Kadden RM, Rounsaville BJ (1997) Validity of the longitudinal, expert, all data procedure for psychiatric diagnosis in patients with psychoactive substance use disorders. Drug Alcohol Depend 45:93-104

33. Young JG, O'Brien JD, Gutterman EM, Cohen P (1987) Research on the clinical interview. J Am Acad Child Adolesc Psychiatry 26:613-620

34. Swets JA (1982) Sensitivities and specificities of diagnostic tests. JAMA 248:548-549

35. Dierker LC et al (2001) Screening for anxiety and depression in early adolescence. J Am Acad Child Adolesc Psychiatry 40:929-936

36. Chmura Kraemer H, Periyakoil VS, Noda A (2002) Kappa coefficients in medical research. Stat Med 21:2109-2129

37. Ivarsson T, Melin K, Wallin L (2008) Categorical and dimensional aspects of co-morbidity in obsessive-compulsive disorder (OCD). Eur Child Adolesc Psychiatry 17:20-31 